\title{
Title: Dissociation of Local and Global Contributions to Detection of Shape with Age
}

\author{
Serena J. Cribb \\ School of Psychology, University of Western Australia \\ Johanna C. Badcock \\ Centre for Clinical Research in Neuropsychiatry, School of Psychiatry and Clinical \\ Neuroscience, University of Western Australia \\ Murray T. Maybery and David R. Badcock \\ School of Psychology, University of Western Australia
}

\begin{abstract}
Author Note
This research was supported by ARC grants to DRB DP1097003 \& DP130102580. JCB is supported by the Medical Research Foundation (Infrastructure Grant), Perth and by the Cooperative Research Centre - Mental Health, Carlton, Victoria. MM’s research group is supported by ARC grant DP120104713. The authors do not have a financial relationship with any commercial entity that has a financial interest in this manuscript.

Thank you to Allison Fox for her assistance with data collection and to Allison McKendrick, David Lawson and Andrew Turpin for their ongoing support of the PsyPad software. Thank you also to Robert Green for his assistance with modelling of probability summation under the Signal Detection Theory model. We would also like to thank all our participants and, where relevant, their parents and guardians.
\end{abstract}

Corresponding Author: Serena J. Cribb 
M304, School of Psychology

University of Western Australia

35 Stirling Hwy, Crawley

Perth WA 6009

AUSTRALIA

Tel: (+61 8) 64883281

Email: serena.cribb@research.uwa.edu.au 


\begin{abstract}
Radial Frequency (RF) patterns are a useful stimulus for assessing sensitivity to changes in shape. With these patterns it is possible to separate sensitivity to local curvature information from the ability to globally integrate information around the contour. Previous work has demonstrated that young, school-aged children are less sensitive to deformation in RF patterns than adults. However, since the efficiency of contour integration was not assessed age-related differences in performance could arise from either changes in the strength of global pooling of information, the sensitivity to local curvature information, or both. In this study, psychophysical methods were used to reassess changes in sensitivity to RF patterns, separating changes in sensitivity to local curvature information from changes in contour integration strength. Typically developing observers (aged 6-24, $\mathrm{N}=104$ ) were tested using a 2-alternative forced-choice discrimination task with either one, two or three cycles of sinusoidal modulation in a pattern of fixed RF. Thresholds were lower for older observers but the rate of change, as more modulation cycles were added, was approximately constant. The results indicate this is due to changes in local curvature sensitivity and not to the strength of contour integration.
\end{abstract}

Keywords: visual development; shape processing; radial frequency patterns; global integration; local sensitivity 
Dissociation of Local and Global Contributions to Detection of Shape with Age Perception of the shape of contours is necessary to break a complex visual scene down into discrete objects, an ability that is required for most tasks that involve vision. Observers are highly sensitive to subtle changes in the shape of contours even when those paths are composed of separated Gabor patches embedded in noise (Field, Hayes, \& Hess, 1993), though they are often more sensitive to closed than incomplete contours (Kovacs \& Julesz, 1993).

While contour detection may seem a simple process, it requires input from a number of different mechanisms. Though some of the relevant visual processes such as visual acuity (Dobson \& Teller, 1978) mature in the first year of life, other functions critical to contour detection have been found to continue to develop into adolescence and early adulthood. For example, the ability to integrate such contour elements across space is poorer in children than adults (Kovács, 2000; Kovacs, Kozma, Feher, \& Benedek, 1999) as is integration of a target contour with its context (Kaldy \& Kovacs, 2003). In adult observers, when collinearity of contour elements is high, proximity of spaced contour elements is unimportant for contour integration, however proximity of contour elements is important for contour integration when collinearity is low; For children, however, spatial proximity of contour elements limits the extent of contour integration regardless of collinearity (Hadad, Maurer, \& Lewis, 2010b). Thus the early stages of processing elements of a contour are driven by the manner in which the contour is displayed, and performance can vary depending on the developmental maturity of the required processes. For example, sensitivity to luminance-defined contours has been shown to reach adult levels at age 9, while sensitivity to subjective contours did not reach adult levels until age 12 (Hadad, Maurer, \& Lewis, 2010a). These variations may be used, in turn, to help separate the sub-processes underpinning shape perception. Following the early processes which detect the properties of the local contour elements, such as position, contrast 
polarity, orientation, width, comes a second stage which globally pools curvature features around shapes (Loffler, 2015; Wilson \& Wilkinson, 2014). This property of contour processing has been proposed following a range of studies on adult expert psychophysical observers but may also be dissociable through differential changes with age (Perreault, Habak, Lepore, \& Bertone, 2013; Wang, Morale, Cousins, \& Birch, 2009). Radial Frequency (RF) patterns (Wilkinson, Wilson, \& Habak, 1998) are a stimulus class that has been employed to measure the ability to integrate curvature information around contours. Two metrics of performance can be derived from their use: sensitivity to local curvature information, and ability to integrate this information together to form a global percept of shape (Almeida, Dickinson, Maybery, Badcock, \& Badcock, 2014). Previous studies (Perreault et al., 2013; Wang et al., 2009) have found children are less sensitive to shape changes in RF patterns than adults, however, these studies did not differentiate between aspects of performance driven by detection of the local contour features and those reflecting the ability to pool this information around the contour. In the present study, we ask whether these two stages of processing are separable by monitoring how both change with chronological age. This knowledge will help localise the level of the visual system most likely to be responsible for the previously reported changes in shape sensitivity with age.

We now present the current view of the critical stages of shape processing. The human visual system processes shape in the ventral stream. The traditional view suggests that the ventral stream is organised in a hierarchical manner with increasingly complex analysis occurring as the level of this hierarchy increases (Felleman \& Van Essen, 1991; Wilson \& Wilkinson, 2015), beginning in area V1 and terminating in the inferotemporal cortex. However, more recent work (for reviews, see Grinter, Maybery, \& Badcock, 2010; Kravitz, Saleem, Baker, Ungerleider, \& Mishkin, 2013) suggests the ventral stream is actually a complex network made up of multiple feedforward and feedback projections to at least 6 
cortical and subcortical regions with distinct functions. The earliest stages of the ventral network process information across small areas of space and are sensitive to local contour information such as the orientation of line fragments (Hubel \& Wiesel, 1968). At this early level, there is some limited pooling of information across receptive fields (Field et al., 1993; Gilbert, Das, Ito, Kapadia, \& Westheimer, 1996; Polat \& Sagi, 1994), however, it is insufficient to account for perception of whole shape contours (Loffler, 2008, 2015). In order to account for this kind of visual performance, the visual system must contain a mechanism that integrates local orientation and curvature information around shape contours. Such integration of local orientation information around shape contours is believed to occur at an intermediate stage of the ventral network, area V4 in monkeys (Pasupathy \& Connor, 1999) and a functionally homologous area in humans (Bowden, Dickinson, Fox, \& Badcock, 2015; Gallant, Shoup, \& Mazer, 2000; Wilkinson et al., 2000). The process of integrating information around contours has been referred to as global processing in many papers in the literature. Given the term "global processing” is used to refer to a number of distinct processes in different areas of the literature (e.g. perception of whole complex images in the Embedded Figures Test literature (Happé \& Frith, 2006) or the detection of the large letters in Navon Figures (Navon, 1977), here, we use the term "global contour integration” for clarity to indicate that we are referring to a particular property of visual form processing.

RF patterns have been used to demonstrate global integration of contour information (Loffler, Wilson, \& Wilkinson, 2003; Wilkinson et al., 1998). These patterns are created by sinusoidally deforming the radius of a circle, resulting in smooth bumps on the contour (Figure 1a). The number of repetitions of the sinusoidal modulator required to fill $360^{\circ}$, its frequency, determines the shape of the pattern. For example, a pattern with a frequency of 3 (RF3) when fully deformed has three bumps and appears similar to a triangle in shape when 
the modulation amplitude is appropriate; an RF4 can appear similar to a square, RF5 a pentagon and so on (see Figure 1b).

\section{Please Insert Figure 1}

The amplitude (A in Eq 1 and Figure 1a) of the sinusoid determines the amount by which the contour of the circle is deformed (i.e. how pronounced the bumps on the contour are), with larger amplitudes leading to a pattern that is easier to discriminate from circular (see Figure 1c). An observer's sensitivity to an RF pattern has typically been obtained by determining the amplitude needed to reliably discriminate the pattern from an unmodulated circle (shown in Figure 1c, far left).

RF patterns can be constructed with any number of cycles of a fixed wavelength of the sinusoid up to the number required to deform the entire contour of the pattern (see Figure 1d). It can be seen in Figure 1d that patterns with more cycles of deformation contain more local curvature information differing from that found in the circle. This means that if observers were using local features alone to make a shape judgement, they would have a greater chance of making a correct judgement as more cycles are added. In order to account for this, previous studies have calculated a probability summation estimate of the improvement that would be expected from using local cues alone to make a shape judgement as cycles are added to an RF pattern. In previous studies the conventional method employed for estimating probability summation has been to take the reciprocal of the average slope of the psychometric function (-1/Q, where $\mathrm{Q}$ is an estimate of psychometric function slope.) following Quick (1974), Loffler et al. (2003) and Bell and Badcock (2008). If the obtained improvement in detection of deformation significantly exceeds that predicted by probability summation, the shape is shown to be processed globally. RF patterns with frequencies between 3 and 8-10 have been shown to be processed globally using this metric, while 
frequencies outside of this range are processed locally (Bell, Badcock, Wilson, \& Wilkinson, 2007; Bell, Dickinson, \& Badcock, 2008; Hess, Wang, \& Dakin, 1999; Jeffrey, Wang, \& Birch, 2002; Loffler et al., 2003; Tan, Dickinson, \& Badcock, 2013; Wilson \& Wilkinson, 1998). Those with frequencies exceeding $\sim 8$ that do not show global processing have results matching the estimate of probability summation (Loffler et al., 2003). Recently, it has been argued that probability summation would be better estimated using a derivation based on signal detection theory (Baldwin, Schmidtmann, Kingdom, \& Hess, 2016; Kingdom, Baldwin, \& Schmidtmann, 2015). The current study will provide both estimates to determine whether the conclusion of global processing at low RFs is still warranted.

Two studies have investigated how sensitivity to fully modulated RF patterns improves across development but neither have dissociated local contour processing stages from global integration stages of shape processing. Both of these studies reported that primary school aged children (aged 7-12) are less sensitive to deformation in complete RF patterns than adults (Perreault et al., 2013; Wang et al., 2009), suggesting that one or more visual processes involved in detection of these patterns is still maturing within this age range. Wang et al. (2009) tested a total of 236 observers aged $0.25-78$ years, concluding that thresholds did not reach the adult mean until age 21, but that some children had sensitivity in the adult range by age 7 . That thresholds asymptote at the age of 21 suggests that some visual processes underlying sensitivity to RF patterns may continue to develop into early adulthood.. It is important to note that in Wang et al's (2009) study, children younger than 2 years of age responded to an RF6 pattern, while older children and adults responded to an RF8 pattern. This use of differing stimuli may have impacted on results, as thresholds have been shown to vary with RF number, being lower for higher RF patterns (Dickinson, McGinty, Webster, \& Badcock, 2012; Hess et al., 1999; Rajeev, Tan, Liyana, \& Metha, 2015; Wilkinson et al., 1998). When comparing the local orientations in RF patterns and 
circles at a particular amplitude, the differences vary with RF number, being greater for RF8 than RF6. The size of the local orientation difference seems to be a critical cue for threshold detection of deformation (Dickinson et al., 2012) meaning observers obtain lower thresholds for RF8 patterns than RF6 patterns. The use of a lower frequency pattern for younger observers may therefore have impacted on the developmental curve obtained by Wang et al. (2009).

Perreault et al. (2013) tested observers who fell into three different age groups: children (7-12 years), adolescents (13-17 years) and adults (18-26 years). Observers were required to detect an RF3 and an RF5, which are known to be globally processed frequencies (Bell \& Badcock, 2008; Jeffrey et al., 2002; Loffler et al., 2003; Tan et al., 2013), and an RF10, which is believed to be processed only locally though some research has suggested global pooling may extend this far (Loffler et al., 2003). The authors reported that adolescents and adults were more sensitive to the shape in RF3 pattern than children, while the groups did not differ in their sensitivity to the RF10 pattern. On this basis, the authors suggested that sensitivity to local curvature information is adult-like in primary school aged children, while global contour integration is still developing.

These previous studies have not measured sensitivity to RF patterns of fixed frequency but with different numbers of cycles, meaning they have not fit an integration slope to examine the extent of global pooling of contour information. The changes reported could be due to changes in effectiveness of global contour integration, changes in sensitivity to local curvature information or changes in both of these factors across this age range. In the present study, the aim is to first determine whether patterns are globally integrated, comparing the previously used probability summation estimates with those based on signal detection theory, arising from more recent considerations. The study will then directly test whether the change in sensitivity to RF patterns across development is due to an 
improvement in local contour sensitivity or global contour integration, or both. A significant relationship between age and steepness of integration slope would indicate that global contour integration is still developing in children. A significant relationship between thresholds for detection of RF patterns and age, in the absence of a relationship between global contour integration and age, would suggest that it is sensitivity to local curvature information that is still developing. A significant relationship between age and both sensitivity to local curvature information and steepness of integration slope would suggest both of these factors are still developing in this age band.

\section{Method}

\section{Observers}

Ethics approval for this project was obtained from the University of Western Australia (UWA) Human Research Ethics Committee. All observers, including children, gave signed informed consent. For participants younger than 17 years old, parents or guardians were encouraged to discuss possible participation with their child first and also gave signed informed consent for their child to take part in the study.

Observers were recruited through one of the following sources at UWA: Children's Activity Program, enrolment in an undergraduate psychology unit, the School of Psychology Community Research Participation Pool or the Guild Volunteering website. One hundred and four observers, aged 6-24 years, with normal or corrected-to-normal visual acuity (6/6 acuity as assessed by Snellen chart) participated voluntarily in the study. The average IQ of this sample, assessed using the Wechsler Abbreviated Scale of Intelligence - Second Edition (WASI-II) (Pearson Assessment, 2011), (M = 109.8, SD = 12.88), was slightly above that of the published norms.

\section{Apparatus}


Stimuli were created using custom software written in MatLab 7.9.0. Stimulus presentation was controlled using the PsyPad application software (Turpin, Lawson, \& McKendrick, 2014) and stimuli were displayed on an Apple iPad Air with a resolution of 2048 x 1536 pixels, a maximum contrast of 90\%, a mean background luminance of 81.83 $\mathrm{cd} / \mathrm{m}^{2}$ (CIE1931 $2^{\circ}$ chromaticity coordinates: $\mathrm{x}=0.314, \mathrm{y}=0.333$ ), a maximum luminance of $340.27 \mathrm{~cd} / \mathrm{m}^{2}$ and a frame rate of $60 \mathrm{~Hz}$.

\section{Stimuli}

Stimuli were RF3 patterns. A frequency of 3 was chosen for two main reasons. Firstly, an RF3 results in a steeper integration slope than RF patterns of higher frequencies (Loffler et al., 2003) thus allowing more room for individual variation in the range indicating global processing. Secondly, fewer thresholds need to be measured for a complete integration function when only 3 cycles of modulation fully modulate the shape and so the testing load on participants is reduced.

The radius of an RF pattern $(R)$ at a given angle $(\theta)$ is given by the formula:

$$
\mathrm{R}(\theta)=\mathrm{R}_{\text {mean }}(1+\mathrm{A} \sin (\omega \theta+\varphi))
$$

In this formula, $R_{\text {mean }}$ is the mean radius of the pattern $\left(1.5^{\circ}\right.$ of visual angle), $\omega$ is the radial frequency (i.e. the frequency of the sine wave used to modulate the pattern, which was always 3 for this study), $\varphi$ is used to control the rotation (or phase) of the pattern which was randomised so the position of the lobes of the pattern was varied from trial to trial, and $\mathrm{A}$ is the amplitude of the modulation which was varied to estimate threshold. The mean radius was chosen following previous work from our research group (Grinter, Maybery, Pellicano, Badcock, \& Badcock, 2010). Sensitivity to RF patterns has been shown to be size invariant within this range, (Wilkinson et al., 1998) so it is unlikely to have impacted on results. 
We developed RF3 patterns with one, two and three cycles of modulation (see Figure 1d). A fully modulated RF3 pattern contains three cycles in $360^{\circ}$ ( $2 \pi$ radians). For patterns with fewer than three cycles of modulation, the transition between the modulated and circular parts of the pattern was smoothed using a profile matching the first derivative of a Gaussian (D1), following earlier authors (Dickinson et al., 2012; Loffler et al., 2003), to prevent the transition from serving as a distinctive local cue. RF patterns had a luminance profile defined by a Gaussian function with a full width at half maximum contrast of 5.62' of visual angle.

Each of the three stimuli was tested at nine amplitudes (see Figure 2), presented in random order using Method of Constant Stimuli, with 15 trials per amplitude completed by each observer, such that each observer completed $15 \times 9 \times 3$ trials in total throughout the experiment. The three stimuli were presented in three separate blocks (one block for each number of cycles) that were completed in counterbalanced order across participants. Amplitudes were chosen to cover the full psychometric function based on the results of prior studies which used similar stimuli and on the results of pilot testing a range of amplitudes on children, adolescents and adults.

\section{Please Insert Figure 2}

\section{Procedure}

A two-spatial-alternative forced-choice procedure was used in which two patterns, one deformed from circular and one a circle, were presented simultaneously side by side on the screen. Stimuli had a mean radius of $1.5^{\circ}$ and a centre-to-centre separation of $3.75^{\circ}$ following Grinter, Maybery, Pellicano, et al. (2010). Each trial was presented for 500ms.

Observers viewed stimuli in a seated position in a darkened room from a distance of $34.5 \mathrm{~cm}$ such that each pixel subtended one minute of visual angle. Where testing was conducted in the lab, viewing distance was maintained using a chin rest. For 12 participants 
who completed testing at their homes, viewing distance was measured using a tape measure, and participants were instructed to maintain viewing at that distance and not to move closer or further away from the screen. The experimenter remained in the room throughout testing in order to ensure these instructions were followed. However, should any minor variation have occurred (e.g. if observers adjusted their posture during a trial), we do not expect that this would have impacted substantially on results given thresholds for detection of RF patterns are robust to size variation (Bell et al., 2008; Wilkinson et al., 1998).

Observers were informed that they would see two shapes on the screen and instructed to indicate the one that looked as though it was "squashed" or deformed. For younger observers, the task was presented in the form of a game in which the deformed circles were meteors that they had to stop by spotting them. To ensure that observers (younger observers in particular) understood the requirements of the task, they were shown an example of a suprathreshold stimulus and asked to indicate which of the two shapes they would choose. All observers responded correctly to this question.

Observers made responses by pressing 'buttons' (highlighted squares) on the touch screen of the iPad that read "first" (on the left hand side) and "second" (on the right hand side) below each of the two stimuli. When unsure of which shape was deformed from circular, participants were encouraged to take their best guess. Each participant then completed a brief practice run of the task. Testing took approximately 15-20 minutes depending on response times. Participants took short breaks at appropriate times between blocks of testing if needed.

\section{Data Analysis}


Raw data were transformed to a percentage of correct responses for each condition. A Quick function (Quick, 1974; Wilson, 1980) was fitted using non-linear regression to each observer's data for each of the three stimulus conditions using the formula:

$$
p(A)=1-2^{\left(-\left(1+\left(\frac{A}{\Delta}\right)^{Q}\right)\right)}
$$

Where $p(\mathrm{~A})$ is the proportion of correct responses at a given amplitude of modulation, and the formula gives $\Delta$, the $75 \%$ correct threshold for stimulus detection, and Q, the slope of the psychometric function. Psychometric functions that could not be fit with a Quick function were not included in the analyses, as this suggests the psychometric function is not well described for these observers. Eighty-eight values for one cycle, 86 values for two cycles and 93 values for 3 cycles met criteria for inclusion.

After fitting the psychometric data for one, two and three cycles for each observer with Equation 2 to obtain $75 \%$ correct thresholds $(\Delta)$, for those observers who had acceptable goodness-of-fit for all three numbers of cycles (71 observers), the inverse relationship between number of cycles and threshold was fitted with a power function to obtain an estimate of the efficiency of global contour integration, following Loffler et al. (2003), using the formula:

$$
Y=k X^{\mathbf{b}}
$$

Where $\mathrm{Y}$ is the $75 \%$ correct threshold, $\mathrm{k}$ is a sensitivity scaler estimating the threshold for a single cycle of modulation on the contour, $\mathrm{X}$ is the number of cycles, and the equation gives $b$, the inverse of $-1 / \mathrm{Q}$ and an estimate of the slope of the contour integration function. Response functions were fitted using nonlinear regression using Prism5 (GraphPad Software, San Diego California USA, www.graphpad.com), to yield best fit estimates for both $\mathrm{k}$ and $\mathrm{b}$. 
The recent work of Kingdom et al. (2015) and Baldwin et al. (2016) , suggests the conventional method of calculating probability summation should be rejected, because it is based on high threshold theory. They propose instead a method for estimating the expected change in thresholds as the number of cycles increase based on signal detection theory. Following their work, we have also estimated thresholds using their methods which are ,outlined in Kingdom and Prins (2016), using the Palamedes toolbox for Matlab (http://www.palamedestoolbox.org/). The data representing percent correct performance were converted to d' (using the Palamaedes function PAL_SDT_2AFC_PCtoDP) to determine psychomteric function parameter which were then used to estimate the thresholds that would arise from probability summation (using PAL_SDT_PS_PCtoSL) on the assumption that observers monitored 120 channels (1 per degree of rotation of the pattern before repetition). Since our patterns had random rotations for each presentation, and thresholds are both rotationally invariant (Bell \& Badcock, 2008) and the feature that is detected at the first stage of processing is the point of maximum difference in orientation from a circle, which is very local (Dickinson et al., 2012), it is reasonable to assume a large number of channels may need to be monitored. Integration slopes were fit to the thresholds for comparison with the data collected from participants. Simulations showed that these slope estimates decrease with increasing assumed channel numbers but asymptote with numbers between 50 to 80 . We can also confirm that with channel number estimates of 3 (1 sample per cycle of the RF3), after outlier removal, the slope estimates for probability summation are still significantly lower than obtained from the data.

\section{Results}

Three metrics of performance are central to our research question: $\Delta$, obtained from Equation 2, the amplitude corresponding to 75\% correct threshold for a given number of cycles of modulation of the RF pattern, Q, the slope of the psychometric function for a given 
number of cycles used to estimate probability summation (either -1/Q or the estimate based on signal detection theory), and b, the integration slope obtained from Equation 3 indicating how thresholds change as more cycles are added to the RF pattern.

A D’Agostino and Pearson omnibus normality test was conducted to test for violations of normality for $75 \%$ correct thresholds for one, two and three cycles of modulation and for integration slope data. Threshold data were not normally distributed for one cycle $(\mathrm{K} 2=51.80, p<.0001)$, two cycles $(\mathrm{K} 2=23.54, p<.0001)$ or three cycles $(\mathrm{K} 2=$ 48.71, $p<.0001)$. However, normalising the data by taking the base-10 logarithmic transform did not change the outcome of any statistical test, so analyses using the raw data are reported. Integration slope data were normally distributed $(\mathrm{K} 2=3.626, p=.163)$. The estimates of $\mathrm{Q}$ for Equation 2 were screened for outliers. Eighteen threshold values (5 for 1 Cycle, 9 for 2 cycles, 4 for 3 cycles, for participants across a range of ages) were identified as outliers, suggesting the psychometric function was not well described for these individuals.

Figure 3 plots the thresholds obtained as a function of age separately for the one, two and three cycle patterns. In order to determine whether performance changes with age the individual thresholds were fit with a power function across age (Motulsky \& Brown, 2006) (see Figure 3). Five outliers were removed from the 1 Cycle condition, 4 outliers were removed from the 2 Cycle condition and 4 outliers were removed from the 3 Cycle condition. The statistics we report here were calculated using the cleaned data with both Q estimate outliers and threshold outliers removed; however, subsequent verification including the outliers did not change the outcome of any statistical test.

The slope of the power function describing the improvement in threshold performance across age was significantly different from zero for RF patterns with one $(F(1,86)=33.62$, $p$ 
$<.0001)$, two $(F(1,89)=24.35, p<.0001)$ and three $(F(1,95)=23.28, p<.0001)$ (see Figure

3) cycles of modulation ${ }^{1}$.

In order to determine whether the integration slope values describing the improvement in threshold as more cycles of modulation were added varied as a function of age of the participants a power function was fitted, using automatic outlier elimination (Motulsky \& Brown, 2006). Graphpad Prism software used an F-Test to determine whether the slope of this function significantly differed from zero (Graphpad Software Inc). No outliers were removed. The index (or slope) of that function did not significantly differ from zero, $F(1,81)=1.02, p=.32$ ) as the slopes did not change with age (see Figure 4 which clearly indicates that while the curves change in vertical location with age it does not change in slope.).

Descriptive statistics are presented in Table 1.

\section{Please Insert Table 1}

Average Q values were 2.19 for 1 cycle (95\% CI 1.85 - 2.55), 2.40 for 2 cycles (95\% CI 2.0 - 2.79) and 2.63 for 3 cycles (95\% CI 2.30 - 2.96) of modulation, yielding probability summation predictions $(-1 / \mathrm{Q})$ of -0.46 , if based on the performance with only one cycle-see Figure 5c, or, -0.41 , if based on the average of all three Q values, as is common (Loffler et al., 2003). An F-test demonstrated that the average slope of the integration function $(\mathrm{M}=$ $0.78,95 \% \mathrm{CI}=-0.71$ to -0.84$)$ was significantly steeper than this prediction, $F(1,81)=$ 7.63, $p=.0071$, thus demonstrating that on average, participants were globally integrating information around the shape contour rather than relying on local contour information to make shape judgements. The same outcome was obtained when comparing the probability summation estimates obtained using the method based on signal detection theory. The mean slope was -0.882 (95\% CI -0.77 to -0.89 , see Figure $5 b)^{2}$. 
Together, these data suggest younger observers were less sensitive to RF3 patterns with any given number of cycles of modulation. However, there was no significant relationship between age and steepness of integration slope, suggesting global contour integration is adult-like in primary school aged children (see Figure 4). The relationship between age and threshold is shown in the bar graphs in Figure 3 where participants are split up into age bands 6-8 $(\mathrm{N}=13), 8-10(\mathrm{~N}=25), 10-12(\mathrm{~N}=14), 14-16(\mathrm{~N}=10), 16-18(\mathrm{~N}=$ 14), $18-20(\mathrm{~N}=23)$, and 20-24 $(\mathrm{N}=5)$. Note the final band covers 4 years rather than 2 because of small $\mathrm{N}$ in the oldest category. The 18 threshold values that were excluded as outliers from earlier data analysis are not included in these graphs.

\section{Please Insert Figure 3}

\section{Please Insert Figure 4}

\section{Please Insert Figure 5}

Given threshold data are non-normally distributed, a series of Spearman correlations were performed to determine whether there was any relationship between IQ and thresholds for 1 , 2 or 3 cycles of modulation, as these are robust to non-normally-distributed data. IQ was not related to threshold for one cycle of modulation $(r=.09, p=.40), 2$ cycles of modulation $(r=-.1, p=.36)$ nor 3 cycles of modulation $(r=-0.07, p=.49$. A Pearson's correlation also demonstrated that IQ was not related to integration slope ( $r=-.2 p=.09$ ).

\section{Discussion}

The aims of the study were first to determine whether contour information is globally integrated with RF patterns, as has previously been assumed, but recently thrown into doubt with concern being raised about a common test for global processing. Secondly this study aimed to to determine whether changes in sensitivity to RF patterns as a function of age are due to improvements in sensitivity to local curvature information or to increased efficiency of 
integration of that information around contours, or both of these processes. The data indicate that all participants showed improvements in threshold as more cycles of deformation were added and the improvements were larger than those predicted by probability summation (using either method for this calculation), indicating global contour integration is occurring and that it occurs at all ages. The data suggest sensitivity to deformation in globally processed RF patterns improves from primary school age to early adulthood for samples whose cognitive ability is in the normal range, consistent with data reported by Wang et al. (2009) and Perreault et al. (2013). However, these changes in sensitivity to RF patterns across development cannot be accounted for by increased effectiveness of global contour integration, as our data suggests there is no improvement in the steepness of integration slopes across this period of development. This shows that the benefit obtained from adding more cycles of modulation to an RF pattern is no greater for adults than it is for children, that is, their ability to integrate information around the contours is equivalent.

The improvement in sensitivity to these patterns across development must therefore be accounted for by some other factor. Our method allows the dissociation of sensitivity to local contour deformations from the integration of that information and the results lead to the suggestion that sensitivity to the local features of the RF contour such as curvature information is, instead, improving as a function of age. This is contrary to the conclusions of Perreault et al. (2013), whose data suggested global processing was not yet mature in primary-school aged children but that sensitivity to local contour information was mature in this age group. The authors reached this conclusion on the basis that children were less sensitive to a fully-modulated RF3 (a globally processed frequency) than adults, but groups were equally sensitive to a fully-modulated RF10 (a locally processed frequency). However, the thresholds reported for each of these groups for the RF10 pattern were approximately at the lowest (and therefore most difficult to detect) amplitude tested (.001) and thus suggestive 
of a ceiling effect. It is possible that if lower amplitudes had been included in testing, children may not have had equivalent thresholds to adults. In future research, it would be important to investigate whether, by including some lower amplitude RF10 patterns, a difference could be found in sensitivity to these patterns between children and adults.

Change in thresholds in the current study cannot be explained by changes in sensitivity to spatial frequency information. A Gaussian luminance profile was chosen because it was expected that its included low spatial frequency range would be better detected at all ages than the fourth-derivative of a Gaussian luminance profile centred on a higher spatial frequency (Bradley \& Freeman, 1982; Ellemberg, Lewis, Hong Liu, \& Maurer, 1999) which is more frequently used in RF pattern studies. Here, we were interested in dissociating local and global contributions to shape perception as age varied and did not want this to be confounded by change in sensitivity to the range of the contained spatial frequency information.

Cognitive factors that show some improvement through childhood and adolescence such as memory (Gathercole, 1998) and attention (Trick \& Enns, 1998) might have an impact on thresholds for detection of RF patterns on a time-limited forced-choice task such as this. However, we believe that the side-by-side stimulus presentation method we used in this study following Grinter et al. (2009) should minimise the impact of these cognitive factors on performance.

A limitation of this study is that we did not have any participants between the ages of 12 and 14 years. We feel it is unlikely that the general pattern of results would be changed with the addition of this age band to the sample, as there seems to be a smooth change in threshold with age in our data. 
There are practical implications of this work for the study of Autism Spectrum Disorder (ASD) and the broader autism phenotype. Children and adolescents with a diagnosis of ASD have been found to show reduced sensitivity to continuous contour RF3 patterns but equivalent sensitivity to RF10 patterns compared to typically developing controls (Grinter, Maybery, Pellicano, et al., 2010; Perreault, Habak, Lepore, Mottron, \& Bertone, 2015). However, research examining neurotypical adults with high levels of autism-like traits indicates that these individuals show equivalent detection thresholds but superior global contour integration for RF3 patterns than those low in autism-like traits (Almeida et al., 2014). This difference between studies may reflect either an improvement in sensitivity to local contour information across development or a difference in sensitivity to local curvature information between individuals with ASD and neurotypical individuals with high levels of autism-like traits. As the current study suggests sensitivity to local contour information does appear to continue developing throughout late childhood and adolescence, it is plausible that individuals with a clinical diagnosis of ASD may have higher thresholds for detection of RF3 patterns as children but improve to a point where they are equivalent to their typically developing peers in adulthood. We are currently conducting work investigating this possibility.

In summary, we present here data suggesting that the local and global contributions to shape perception may be dissociated and that changes in sensitivity to RF patterns across development are due to improvements in sensitivity to local curvature information and not global contour integration. This finding has implications for higher-order shape processing as well as for changes in sensitivity to shape information across development in ASD. 


\section{References}

Almeida, R. A., Dickinson, J. E., Maybery, M. T., Badcock, J. C., \& Badcock, D. R. (2014). Enhanced global integration of closed contours in individuals with high levels of autistic-like traits. Vision Research, 103, 109-115. doi: 10.1016/j.visres.2014.08.015

Baldwin, A. S., Schmidtmann, G., Kingdom, F. A. A., \& Hess, R. F. (2016). Rejecting probability summation for RF patterns, not so Quick! Vision Research, pii: S0042-6989(16)00044-4. doi: 10.1016/j.visres.2016.03.003

Bell, J., \& Badcock, D. R. (2008). Luminance and contrast cues are integrated in global shape detection with contours. Vision Research, 48(21), 2336-2344. doi: 10.1016/j.visres.2008.07.015

Bell, J., Badcock, D. R., Wilson, H., \& Wilkinson, F. (2007). Detection of shape in radial frequency contours: Independence of local and global form information. Vision Research, 47(11), 15181522. doi: 10.1016/j.visres.2007.01.006

Bell, J., Dickinson, J. E., \& Badcock, D. R. (2008). Radial frequency adaptation suggests polar-based coding of local shape cues. Vision Research, 48(21), 2293-2301. doi: 10.1016/j.visres.2008.07.003

Bowden, V. K., Dickinson, J. E., Fox, A. M., \& Badcock, D. R. (2015). Global shape processing: A behavioral and electrophysiological analysis of both contour and texture processing. Journal of Vision, 15(13), 18-18. doi: 10.1167/15.13.18

Bradley, A., \& Freeman, R. D. (1982). Contrast sensitivity in children. Vision Research, 22(8), 953-959. doi: 10.1016/0042-6989(82)90031-1

Dickinson, J. E., McGinty, J., Webster, K. E., \& Badcock, D. R. (2012). Further evidence that local cues to shape in RF patterns are integrated globally. Journal of Vision, 12(12), 16-16. doi: 10.1167/12.12.16

Dobson, V., \& Teller, D. Y. (1978). Visual acuity in human infants: a review and comparison of behavioral and electrophysiological studies. Vision Research, 18(11), 1469-1483. doi: 10.1016/0042-6989(78)90001-9

Ellemberg, D., Lewis, T. L., Hong Liu, C., \& Maurer, D. (1999). Development of spatial and temporal vision during childhood. Vision Research, 39(14), 2325-2333. doi: 10.1016/S00426989(98)00280-6

Felleman, D. J., \& Van Essen, D. C. (1991). Distributed hierarchical processing in the primate cerebral cortex. Cerebral Cortex, 1(1), 1-47. doi: 10.1093/cercor/1.1.1

Field, D. J., Hayes, A., \& Hess, R. F. (1993). Contour integration by the human visual system: Evidence for a local "association field". Vision Research, 33(2), 173-193. doi: 10.1016/00426989(93)90156-Q

Gallant, J. L., Shoup, R. E., \& Mazer, J. A. (2000). A human extrastriate area functionally homologous to macaque V4. Neuron, 27(2), 227-235. doi: 10.1016/S0896-6273(00)00032-5

Gathercole, S. E. (1998). The development of memory. Journal of Child Psychology and Psychiatry, 39(1), 3-27. doi: 10.1111/1469-7610.00301

Gilbert, C. D., Das, A., Ito, M., Kapadia, M., \& Westheimer, G. (1996). Spatial integration and cortical dynamics. Proceedings of the National Academy of Sciences, 93(2), 615-622. doi: 10.1073/pnas.93.2.615

Graphpad Software Inc. Is the slope significantly different than zero? Retrieved 28/02/2016, from http://www.graphpad.com/guides/prism/6/curvefitting/index.htm?istheslopesignificantlydifferentthanzero .htm

Grinter, E. J., Maybery, M. T., \& Badcock, D. R. (2010). Vision in developmental disorders: is there a dorsal stream deficit? Brain research bulletin, 82(3), 147-160.

Grinter, E. J., Maybery, M. T., Pellicano, E., Badcock, J. C., \& Badcock, D. R. (2010). Perception of shapes targeting local and global processes in autism spectrum disorders. Journal of Child Psychology \& Psychiatry, 51(6), 717-724. doi: 10.1111/j.1469-7610.2009.02203.x 
Grinter, E. J., Maybery, M. T., Van Beek, P. L., Pellicano, E., Badcock, J. C., \& Badcock, D. R. (2009). Global visual processing and self-rated autistic-like traits. Journal of Autism \& Developmental Disorders, 39(9), 1278-1290. doi: 10.1007/s10803-009-0740-5

Hadad, B., Maurer, D., \& Lewis, T. L. (2010a). The development of contour interpolation: Evidence from subjective contours. Journal of Experimental Child Psychology, 106(2-3), 163-176. doi: 10.1016/j.jecp.2010.02.003

Hadad, B., Maurer, D., \& Lewis, T. L. (2010b). The effects of spatial proximity and collinearity on contour integration in adults and children. Vision Research, 50(8), 772-778. doi: 10.1016/j.visres.2010.01.021

Happé, F., \& Frith, U. (2006). The weak coherence account: Detail-focused cognitive style in autism spectrum disorders. Journal of Autism and Developmental Disorders, 36(1), 5-25. doi: 10.1007/s10803-005-0039-0

Hess, R. F., Wang, Y.-Z., \& Dakin, S. C. (1999). Are judgements of circularity local or global? Vision Research, 39(26), 4354-4360. doi: 10.1016/S0042-6989(99)00153-4

Hubel, D. H., \& Wiesel, T. N. (1968). Receptive fields and functional architechture of monkey striate cortex. The Journal of Physiology, 195, 215-243. doi: 10.1113/jphysiol.1968.sp008455

Jeffrey, B. G., Wang, Y.-Z., \& Birch, E. E. (2002). Circular contour frequency in shape discrimination. Vision Research, 42(25), 2773-2779. doi: 10.1016/S0042-6989(02)00332-2

Kaldy, Z., \& Kovacs, I. (2003). Visual context integration is not fully developed in 4-year-old children. Perception, 32(6), 657-666. doi: 10.1068/p3473

Kingdom, F. A. A., Baldwin, A. S., \& Schmidtmann, G. (2015). Modeling probability and additive summation for detection across multiple mechanisms under the assumptions of signal detection theory. Journal of Vision, 15(5), 1-1. doi: 10.1167/15.5.1

Kingdom, F. A. A., \& Prins, N. (2016). Psychophysics: A practical introduction, 2nd edition: Academic Press, an imprint of Elsevier.

Kovács, I. (2000). Human development of perceptual organization. Vision Research, 40(10-12), 13011310. doi: 10.1016/S0042-6989(00)00055-9

Kovacs, I., \& Julesz, B. (1993). A closed curve is much more than an incomplete one: Effect of closure in figure-ground segmentation. Proceedings of the National Academy of Sciences, 90(16), 7495-7497. doi: 10.1073/pnas.90.16.7495

Kovacs, I., Kozma, P., Feher, A., \& Benedek, G. (1999). Late maturation of visual spatial integration in humans. Proceedings of the National Academy of Sciences of the United States of America, 96(21), 12204-12209. doi: 10.1073/pnas.96.21.12204

Kravitz, D. J., Saleem, K. S., Baker, C. I., Ungerleider, L. G., \& Mishkin, M. (2013). The ventral visual pathway: An expanded neural framework for the processing of object quality. Trends in Cognitive Sciences, 17(1), 26-49. doi: 10.1016/j.tics.2012.10.011

Loffler, G. (2008). Perception of contours and shapes: Low and intermediate stage mechanisms. Vision Research, 48(20), 2106-2127. doi: 10.1016/j.visres.2008.03.006

Loffler, G. (2015). Probing intermediate stages of shape processing. Journal of Vision, 15(7), 1-1. doi: 10.1167/15.7.1

Loffler, G., Wilson, H. R., \& Wilkinson, F. (2003). Local and global contributions to shape discrimination. Vision Research, 43, 519-530. doi: 10.1016/S0042-6989(02)00686-7

Motulsky, H. J., \& Brown, R. E. (2006). Detecting outliers when fitting data with nonlinear regression - a new method based on robust nonlinear regression and the false discovery rate. $B M C$ Bioinformatics, 7, 123-123. doi: 10.1186/1471-2105-7-123

Navon, D. (1977). Forest before trees: The precedence of global features in visual perception. Cognitive psychology, 9(3), 353-383. doi: 10.1016/0010-0285(77)90012-3

Pasupathy, A., \& Connor, C. E. (1999). Responses to contour features in macaque area V4. Journal of Neurophysiology, 82(5), 2490-2502. 
Perreault, A., Habak, C., Lepore, F., \& Bertone, A. (2013). Investigating the interaction between low and intermediate levels of spatial vision at different periods of development. Journal of Vision, 13(14), 17. doi: 10.1167/13.14.17

Perreault, A., Habak, C., Lepore, F., Mottron, L., \& Bertone, A. (2015). Behavioral evidence for a functional link between low-and mid-level visual perception in the autism spectrum. Neuropsychologia, 77, 380-386. doi: 10.1016/j.neuropsychologia.2015.09.022

Polat, U., \& Sagi, D. (1994). The architecture of perceptual spatial interactions. Vision Research, 34(1), 73-78. doi: 10.1016/0042-6989(94)90258-5

Quick, R. F., Jr. (1974). A vector-magnitude model of contrast detection. Kybernetik, 16(2), 65-67. doi: $10.1007 /$ bf00271628

Rajeev, N., Tan, E., Liyana, R., \& Metha, A. (2015). Shape discrimination thresholds among subjects with emmetropia and corrected myopia. Clinical and Experimental Optometry, 98(4), 353358. doi: 10.1111/cxo.12249

Tan, K. W. S., Dickinson, J. E., \& Badcock, D. R. (2013). Detecting shape change: Characterizing the interaction between texture-defined and contour-defined borders. Journal of Vision, 13(14), 1-16. doi: $10.1167 / 13.14 .12$

Trick, L. M., \& Enns, J. T. (1998). Lifespan changes in attention: The visual search task. Cognitive Development, 13(3), 369-386. doi: 10.1016/S0885-2014(98)90016-8

Turpin, A., Lawson, D. J., \& McKendrick, A. M. (2014). PsyPad: A platform for visual psychophysics on the iPad. Journal of Vision, 14(3), 1-7. doi: 10.1167/14.3.16

Wang, Y. Z., Morale, S. E., Cousins, R., \& Birch, E. E. (2009). Course of development of global hyperacuity over lifespan. Optometry and Vision Science, 86(6), 695-700. doi: 10.1097/opx.0b013e3181a7b0ff

Wilkinson, F., James, T. W., Wilson, H. R., Gati, J. S., Menon, R. S., \& Goodale, M. A. (2000). An fMRI study of the selective activation of human extrastriate form vision areas by radial and concentric gratings. Current Biology, 10(22), 1455-1458. doi: 10.1016/S0960-9822(00)008009

Wilkinson, F., Wilson, H. R., \& Habak, C. (1998). Detection and recognition of radial frequency patterns. Vision Research, 38(22), 3555-3568. doi: 10.1016/S0042-6989(98)00039-X

Wilson, H. R. (1980). A transducer function for threshold and suprathreshold human vision. Biological Cybernetics, 38(3), 171-178. doi: 10.1007/bf00337406

Wilson, H. R., \& Wilkinson, F. (1998). Detection of global structure in Glass patterns: implications for form vision. Vision Research, 38(19), 2933-2947. doi: 10.1016/S0042-6989(98)00109-6

Wilson, H. R., \& Wilkinson, F. (2014). Configural pooling in the ventral pathway. Werner JS Chalupa L.(Eds.) The new visual neurosciences, 617-626.

Wilson, H. R., \& Wilkinson, F. (2015). From orientations to objects: Configural processing in the ventral stream. Journal of Vision, 15(7), 4-4. doi: 10.1167/15.7.4 


\section{Footnotes}

${ }^{1}$ Visual inspection suggested younger participants may have had more variable thresholds, so the coefficient of variation was calculated for each age band to examine this. No systematic difference in the coefficient of variation was seen between age bands.

2. Kingdom and Prins (2016) note that for probability summation, when modelled using signal detection theory, the estimate of Q, the slope of the psychometric function, should decrease as extra signals, or cycles in our case, are added to the RF pattern. A one-way repeated-measures ANOVA (including participants for whom thresholds for each number of cycles were available) suggests that there is no relationship between Q value and number of cycles of modulation $(F(1.95,163.8)=2.18, p=.12)$, which is also inconsistent with the view that the data can be accounted for by probability summation. 
Table 1

Descriptive statistics for thresholds and integration slopes

\begin{tabular}{lccc}
\hline & Mean & 95\% CI Lower & 95\% CI Upper \\
\hline 1 Cycle Threshold & .032 & .028 & .035 \\
2 Cycles Threshold & .020 & .018 & .022 \\
3 Cycles Threshold & .014 & .012 & .015 \\
Integration across cycles & -.777 & -.839 & -.715 \\
\hline
\end{tabular}



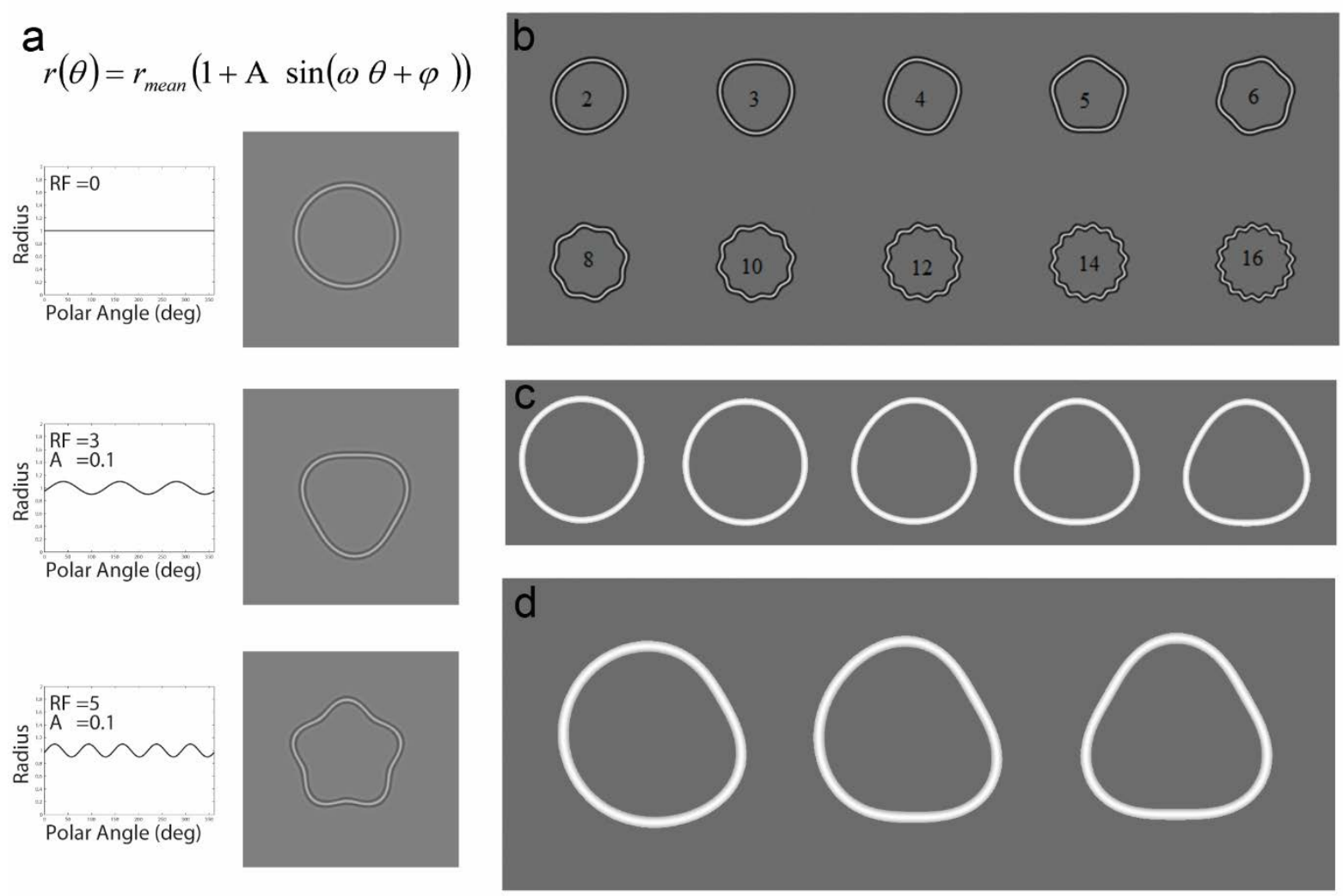

Figure 1. a) From top to bottom, the equation used to create an RF pattern, an unmodulated circle and its polar plot, and an RF3 and an RF5 with their corresponding polar plots. b) Different frequencies of RF patterns (frequency is specified in the centre of each pattern), all with modulation amplitudes (A) of 0.05. c) RF3 patterns at different amplitudes, from left to right: an unmodulated circle (amplitude $=0$ ), amplitudes of .01, .03, .05, .07. d) From left to right, an RF 3 pattern with one, two and three cycles of modulation at an amplitude of .1. The value of $r_{\text {mean }}$ increases in the lower panels on the right to show the size change that results. 
号

Figure 2. Amplitudes tested for observers of different chronological ages for one, two and three cycles of modulation. The mean threshold obtained for observers tested on this range of amplitudes appears to the right of each range of amplitudes with 95\% CIs. 
Figure 3. Bar graphs showing means and 95\% CIs for thresholds for each number of cycles and integration slope by age band. 


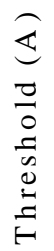

Figure 4. Power function slopes plotted by age band to show the relationship between age, threshold and strength of global integration. Note that the lines systematically change in height, indicating greater sensitivity to the pattern at all numbers of cycles, but do not systematically change in slope. 
Figure 5. The individual integration slope estimates are plotted to show a) the estimates obtained from the participant's data, the probability summation estimates obtained using the signal detection theory based procedure (b) or those obtained using -1/Q (c). 